\title{
Effects of Psychotropic Drugs on Quantitative EEG among Patients with Schizophrenia-spectrum Disorders
}

\author{
June Hyun ${ }^{1}$, Myung Jae Baik², Ung Gu Kang ${ }^{1,3}$ \\ ${ }^{1}$ Department of Neuropsychiatry, Seoul National University Hospital, Seoul, ${ }^{2}$ The Armed Forces Capital Hospital, Seongnam, ${ }^{3}$ Department \\ of Psychiatry and Behavioral Science, Seoul National University College of Medicine \& Institute of Human Behavioral Medicine, Seoul \\ National University, Seoul, Korea
}

\begin{abstract}
Objective: We examined how psychotropic medications affected quantitative EEG (qEEG) results among patients with a schizophrenia-spectrum disorder.

Methods: The drugs were clustered into nine groups depending on their mechanism. We hypothesized that drugs would affect the relative power shown in GEEG results independently and investigated the effect of each drug group on relative power using multiple linear regression analysis and independent samples t-tests.

Results: We found that antipsychotics other than clozapine induced an increase in the relative power of alpha activity. Clozapine markedly increased slow waves and decreased alpha activity in the occipital area. The main findings for antidepressants and antiepileptic drugs were the beta increment and lithium increased the power of delta and theta activity. However, we found no evident changes in power due to benzodiazepine.

Conclusion: Our results are generally consistent with previous pharmaco-EEG studies, despite some differences. Therefore, the EEG effect in each drug group could be singled out even under the polypharmacy condition, with the possible exception of benzodiazepines. Our results support using a new methodological approach to identify the qEEG effects of various psychotropic drugs in clinical settings
\end{abstract}

KEY WORDS: Pharmaco-EEG; Polypharmacy; Psychotropic drugs; Quantitative EEG; Relative power; Schizophrenia-spectrum disorder.

\section{INTRODUCTION}

A human electroencephalogram (EEG) can be affected by various psychotropic drugs. German physiologist and psychiatrist Hans Berger first described how barbiturates, morphine, and scopolamine affect EEG results in 1933, and researches about how psychotropic drugs affect EEG results are still being conducted. ${ }^{1)}$ The quantitative EEG (qEEG) is a method enabling the quantitative measurement of EEG by transforming the EEG signals into numerical parameters by a mathematical processing called the Fast Fourier Transformation (FFT). ${ }^{2}$ Since the development of the qEEG, many pharmaco-EEG studies have ap-

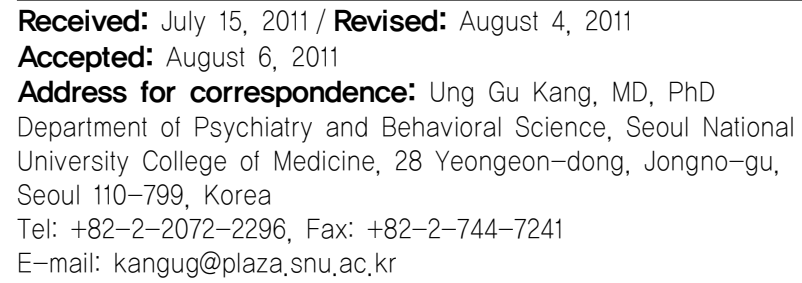

plied this technique. Its potential utility includes assisting new drug development, monitoring adverse effects of medication, selecting appropriate medication, and predicting clinical response to psychotropic drugs, ${ }^{3,4)}$ although it is being used less now due to the development of functional imaging techniques such as fMRI and molecular biology. ${ }^{1)}$

Previous pharmaco-EEG studies of typical antipsychotics have generally produced consistent findings. Sedative low-potency antipsychotics such as chlorpromazine increase slow waves and decrease alpha activity, whereas non-sedative high-potency antipsychotics such as haloperidol have less effect on slow waves but increase alpha and alpha-adjacent beta activity. ${ }^{4}$ A few quantitative pharmaco-EEG studies have been conducted using atypical antipsychotics including risperidone, ${ }^{5}$ olanzapine, ${ }^{6}$ amisulpride, ${ }^{7)}$ and aripiprazole. ${ }^{8)}$ These studies have produced less consistent results than those involving typical antipsychotics. In contrast, previous studies using clozapine, another atypical antipsychotic, have produced 
relatively consistent findings of increased slow waves, despite some controversy about changes in alpha and beta activity, which might be affected by treatment duration and clinical response. ${ }^{9-12)}$

Quantitative pharmaco-EEG studies involving antidepressants have reported qEEG changes induced by the selective serotonin reuptake inhibitors (SSRI) paroxetine, ${ }^{13)}$ sertraline, ${ }^{14)}$ and fluvoxamine. ${ }^{15)}$ Some studies have also been conducted on the qEEG effects of antiepileptic drugs ${ }^{16-18)}$ and lithium, ${ }^{19,20)}$ which are commonly used as mood stabilizers in psychiatric clinical settings.

Most previous research about pharmaco-EEGs has involved placebo-controlled studies after the administration of a single test dose of medication in healthy adults or patients with the same disease, such as schizophrenia. These studies are limited by their small sample size, usually around 20 subjects, and their acute administration of only a single drug. Thus, these results are difficult to apply in a real clinical setting where most patients are chronically medicated with mixtures of psychotropics.

In this study, we investigated the effects of psychotropic drugs on qEEG results among patients who had been diagnosed with a schizophrenia-spectrum disorder. We included a sufficiently large sample size and all the psychotropic drugs administered to subjects.

\section{METHODS}

\section{Study Subjects}

Patients aged between 15 and 65 years who had undergone a qEEG at the Seoul National University Hospital qEEG lab between January 2009 and June 2010 were screened for a diagnosis of a schizophrenia-spectrum disorder (i.e., schizophrenia, schizophreniform disorder, schizoaffective disorder, brief psychotic disorder, delusional disorder, psychotic disorder due to general medical condition, or psychotic disorder NOS) according to DSM-IV criteria. Among a total of 166 possible participants, four were excluded because of poor-quality recording, so the final study population included 162 patients.

\section{qEEG Recordings/Processing}

EEG results were acquired using Neuroscan (Compumedics Ltd., Abbotsford, VIC, Australia) with a sampling rate of $500 \mathrm{~Hz}$ and 24-bit digitization. A band-pass filter with a range of $0.3-30 \mathrm{~Hz}$ was used, and the notch filter was off. Each patient underwent 15 minutes of eye-closed resting EEG testing while seated comfortably in a quiet, dimly lit, temperature-controlled, electromagnetic fieldshielded room. Vigilance was controlled by the EEG technician alerting patients by microphone when signs of drowsiness (e.g., slow lateral eye movement, disappearance of alpha waves in occipital area) were observed during recordings. The international 10-20 electrode system was used to position 21 electrodes (Fpz, Fp1, Fp2, Fz, F3, F4, F7, F8, Cz, C3, C4, Pz, P3, P4, T3, T4, T5, T6, Oz, O1, $\mathrm{O} 2)$ with a mixed-ear reference. To detect artifacts induced by eyeball movement, horizontal and vertical electrooculograms were also recorded. We selected at least 10 non-overlapping pieces of at least $15 \mathrm{sec}$ continuous artifact-free recording by a manual inspection. Data consistency was confirmed by test-retest and split-half correlations $>0.95$ per Neuroguide software (version 2.3.1. Applied Neuroscience, Inc., St. Petersburg, FL, USA). These clean epochs were subjected to a Fast Fourier Transform (FFT) algorithm to calculate absolute power in delta $(1.5-3.5 \mathrm{~Hz})$, theta $(3.5-7.5 \mathrm{~Hz})$, alpha $(7.5-12.5 \mathrm{~Hz})$, and beta $(12.5-25.0 \mathrm{~Hz})$ bands. The relative power of each band was calculated as the fraction of total power including all four bands using the same software.

\section{Drug Treatment}

Any drugs prescribed by psychiatrists were included in the analysis, with the exception of $\mathrm{MgO}$, which is prescribed to relieve constipation. The drugs were clustered into nine groups depending on their clinical use : antipsychotics other than clozapine (AP), clozapine (CLZ), antidepressant (AD), antiepileptic (AED), lithium (Li), benzodiazepine (BDZ), zolpidem (ZPD), anticholinergics

Table 1. Medication groups classified according to the mechanism

\begin{tabular}{cl}
\hline Group name & \multicolumn{1}{c}{ Drugs included } \\
\hline AP & $\begin{array}{c}\text { Risperidone, Quetiapine, Olanzapine, Aripiprazole, } \\
\text { Amisulpride, Paliperidone, Ziprasidone, Haloperidol }\end{array}$ \\
CLZ & Clozapine \\
AD & Escitalopram, Sertraline, Paroxetine, Fluoxetine, \\
& Bupropion, Venlafaxine, Trazodone \\
AED & Valproate, Carbamazepine, Lamotrigine, \\
& Topiramate, Levetiracetam \\
Li & Lithium \\
BDZ & Lorazepam, Clonazepam, Alprazolam, Diazepam \\
ZPD* & Zolpidem \\
AC* & Benztropine, Trihexyphenidyl \\
PPL* & Propranolol \\
\hline
\end{tabular}

$A P$, antipsychotics other than clozapine; CLZ, clozapine; $A D$, antidepressants; AED, antiepileptic drugs; Li, Lithium; BDZ, benzodiazepines; ZPD, zolpidem; AC, anticholinergics; PPL, propranolol.

* Results for these groups as independent variables were not presented. 
(AC), and propranolol (PPL) (Table 1). We singled out clozapine as a separate group because clozapine has distinctive properties compared with other antipsychotics ${ }^{21)}$ and is the most well-known antipsychotics in terms of pharmaco-EEG properties. ${ }^{4)}$ Our analysis did not include medications that are prescribed by clinicians other than psychiatrists to control other general medical conditions (e.g., antihypertensive drugs), but it did include antiepileptic drugs prescribed by neurologists to control epilepsy. We did not analyze the dosage of administered drugs nor the time of drug administration before recording.

\section{Statistics}

We hypothesized that each group of drugs would affect qEEG relative power independently, and conducted a multiple linear regression analysis with the use or non-use of each specific drug group by each individual patient as independent variables. We included all nine groups of medication as independent variables to increase the validity of linear regression. However, we only reported the results for six psychotropic groups (AP, CLZ, AD, AED, $\mathrm{Li}$, and $\mathrm{BDZ}$ ).

To supplement the regression analysis, we conducted a secondary analysis with independent-samples $t$-tests to compare mean relative powers between patients given (or not given) drugs from a specific group. We also conducted an additional analysis for clozapine, which will be described in more detail in the Results section.

The results were presented using probability maps on which squares or circles of different colors were marked according to error probability. All statistical analyses were performed using PASW Statistics 18 (SPSS Inc., Chicago, IL, USA).

\section{RESULTS}

\section{Demographic Characteristics}

Participants had a mean (SD) age of 30.8 (10.53) years. The group included 85 men and 77 women; 152 were right-handed, and 10 were left-handed.

\section{Medication}

Table 2 presents the number of participants in each drug group. Totals exceed the total number of participants because most patients were receiving polypharmacy; we observed a tendency for polypharmacy even among drugs

Table 2. Descriptive statistics for individual drugs

\begin{tabular}{|c|c|c|c|c|c|c|}
\hline Group (subtotal N) & Drug & $\mathrm{N}$ & Min. dose (mg) & Max. dose (mg) & Mean dose (mg) & $\mathrm{SD}$ \\
\hline \multirow[t]{8}{*}{ AP (138) } & Risperidone & 56 & 0.50 & 2.50 & 1.88 & 0.38 \\
\hline & Olanzapine & 25 & 2.50 & 10.00 & 8.50 & 2.80 \\
\hline & Quetiapine & 26 & 25.00 & 400.00 & 213.46 & 135.50 \\
\hline & Aripiprazole & 26 & 5.00 & 15.00 & 10.58 & 4.32 \\
\hline & Ziprasidone & 3 & 80.00 & 80.00 & 80.00 & 0.00 \\
\hline & Haloperidol & 3 & 3.00 & 10.00 & 7.67 & 4.04 \\
\hline & Amisulpride & 11 & 100.00 & 400.00 & 336.36 & 112.01 \\
\hline & Paliperidone & 25 & 3.00 & 9.00 & 6.08 & 2.04 \\
\hline CLZ (47) & Clozapine & 47 & 25.00 & 100.00 & 69.15 & 36.94 \\
\hline \multirow[t]{9}{*}{$A D(36)$} & Escitalopram & 6 & 10.00 & 12.50 & 10.42 & 1.02 \\
\hline & Sertraline & 17 & 50.00 & 50.00 & 50.00 & 0.00 \\
\hline & Paroxetine & 4 & 12.50 & 20.00 & 16.25 & 4.33 \\
\hline & Fluoxetine & 6 & 20.00 & 20.00 & 20.00 & 0.00 \\
\hline & Fluvoxamine & 1 & 50.00 & 50.00 & 50.00 & . \\
\hline & Bupropion & 1 & 150.00 & 150.00 & 150.00 & . \\
\hline & Venlafaxine & 1 & 75.00 & 75.00 & 75.00 & . \\
\hline & Mirtazapine & 2 & 15.00 & 30.00 & 22.50 & 10.61 \\
\hline & Trazodone & 1 & 25.00 & 25.00 & 25.00 & . \\
\hline \multirow[t]{5}{*}{ AED (29) } & Valproate & 21 & 150.00 & 750.00 & 440.48 & 137.49 \\
\hline & Lamotrigine & 8 & 25.00 & 100.00 & 59.38 & 35.20 \\
\hline & Carbamazepine & 2 & 200.00 & 200.00 & 200.00 & 0.00 \\
\hline & Topiramate & 2 & 25.00 & 25.00 & 25.00 & 0.00 \\
\hline & Levetiracetam & 1 & 250.00 & 250.00 & 250.00 & . \\
\hline $\mathrm{Li}(7)$ & Lithium & 7 & 300.00 & 300.00 & 300.00 & 0.00 \\
\hline \multirow[t]{4}{*}{ BDZ (108) } & Lorazepam & 72 & 0.50 & 4.00 & 0.80 & 0.46 \\
\hline & Clonazepam & 37 & 0.50 & 0.50 & 0.50 & 0.00 \\
\hline & Diazepam & 6 & 2.00 & 9.00 & 5.17 & 2.23 \\
\hline & Alprazolam & 8 & 0.25 & 0.50 & 0.28 & 0.09 \\
\hline
\end{tabular}

AP, antipsychotics other than clozapine; CLZ, clozapine; AD, antidepressants; AED, antiepileptic drugs; Li, Lithium; BDZ, benzodiazepines. 
within the same group. Nine patients received no medication. Table 2 also presents the number of participants using individual drugs and their mean dosage.

When subjected to a correlation analysis, group AP showed a statistically significant negative correlation with group CLZ and a significant positive correlation with group $\mathrm{BDZ}$, but no significant correlations appeared between groups CLZ and BDZ. No significant correlations

Table 3. Pearson correlation coefficient between medication groups

\begin{tabular}{|c|c|c|c|c|c|c|c|}
\hline & & AP & CLZ & $A D$ & AED & $\mathrm{Li}$ & $\mathrm{BDZ}$ \\
\hline \multirow[t]{2}{*}{ AP } & Pearson correlation & 1 & $-.277^{\dagger}$ & -.070 & .013 & .089 & $.184^{*}$ \\
\hline & Sig. (2-tailed) & & .000 & .378 & .865 & .262 & .019 \\
\hline \multirow[t]{2}{*}{ CLZ } & Pearson correlation & $-.277^{\dagger}$ & 1 & .059 & .063 & -.066 & .097 \\
\hline & Sig. (2-tailed) & .000 & & .459 & .425 & .400 & .220 \\
\hline \multirow[t]{2}{*}{$A D$} & Pearson correlation & -.070 & .059 & 1 & .138 & -.041 & -.031 \\
\hline & Sig. (2-tailed) & .378 & .459 & & .081 & .608 & .691 \\
\hline \multirow[t]{2}{*}{ AED } & Pearson correlation & .013 & .063 & .138 & 1 & -.099 & .023 \\
\hline & Sig. (2-tailed) & .865 & .425 & .081 & & .209 & .774 \\
\hline \multirow[t]{2}{*}{$\mathrm{Li}$} & Pearson correlation & .089 & -.066 & -.041 & -.099 & 1 & .150 \\
\hline & Sig. (2-tailed) & .262 & .400 & .608 & .209 & & .056 \\
\hline \multirow[t]{2}{*}{$\mathrm{BDZ}$} & Pearson correlation & $.184^{*}$ & .097 & -.031 & .023 & .150 & 1 \\
\hline & Sig. (2-tailed) & .019 & .220 & 691 & .774 & .056 & \\
\hline
\end{tabular}

AP, antipsychotics other than clozapine; CLZ, clozapine; AD, antidepressants; AED, antiepileptic drugs; Li, Lithium; BDZ, benzodiazepines. ${ }^{*}$ Correlation is significant at $p=0.05$ level (2-tailed); ${ }^{\dagger}$ Correlation is significant at $p=0.01$ level (2-tailed).

AP

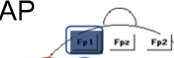

Fif $\mathrm{Fin}_{3}$

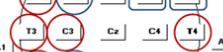

(15) at (02) der delta



Fin $\mathrm{Fa} F \mathrm{Fat}$

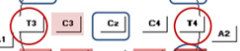

$P_{P_{3}} \mathrm{P}_{2}$ or 02 alpha

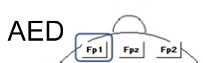

(F)

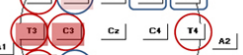

(I5) $P_{3} P_{2}$ o1 (02) der delta

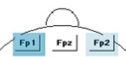

Fis

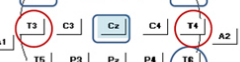

I5] $p_{3}$

an ${ }_{02}$ alpha

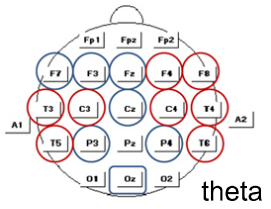

CLZ

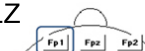

(17)

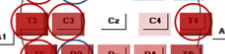

(15) (15) $p_{2} p_{4}$

delta

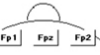

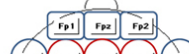

(a) $\mathrm{Fi}$ ( $\mathrm{F}=\mathrm{F}$
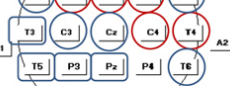

o1 021 on beta

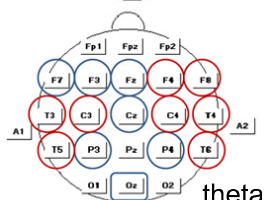

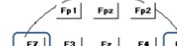

(I) $\mathrm{C}_{2}$

(I3) $\mathrm{C}_{3} \mathrm{C}_{\mathrm{C}} \mathrm{C}_{4} \mathrm{CH}_{\mathrm{A} 2}$



ar 00 alpha

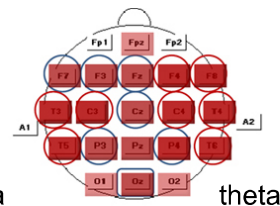

$\mathrm{AD}$

A

(a) $F 31$

(13) $c=c 2$

IV) $P_{3}$ P o1 (02) delta

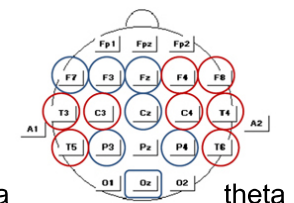

(n)

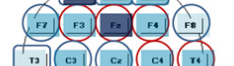

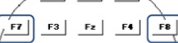

$A_{11} \underbrace{}_{T_{15}}$

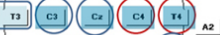

(IS

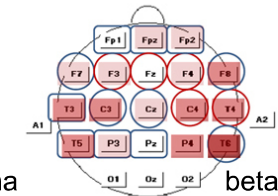

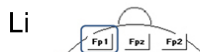

(FI) $\mathrm{F3}$

(a) $c=21$ as (rit)

(I5) $P_{3} P_{2} P_{4}$ or delta

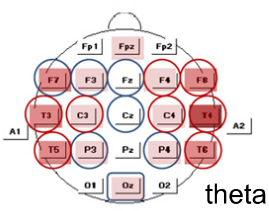

BDZ

a1 02 alpha

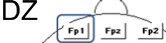

(72) $F 3$

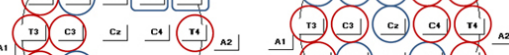

(I5) $P_{3} P_{2}$ or (az) der delta (P3) $P_{2}\left(P_{4}\right.$ (If)
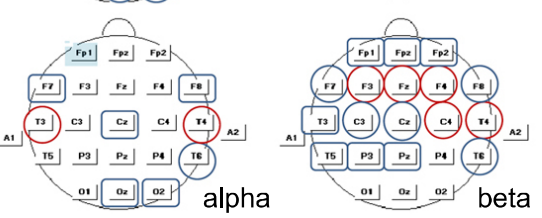

alpha

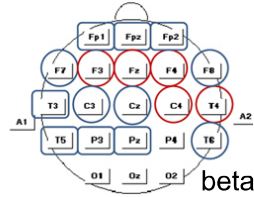

$p$-value in ANOVA

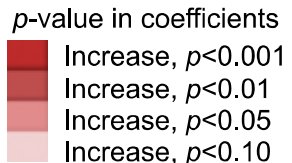

$p<0.10$

Decrease, $p<0.10$

Decrease, $p<0.05$

Decrease, $p<0.01$

Decrease, $p<0.001$

Fig. 1. Statistical probability maps according to multiple regression analysis. AP, antipsychotics other than clozapine; CLZ, clozapine; AD, antidepressants; AED, antiepileptic drugs; Li, Lithium; BDZ, benzodiazepines. 


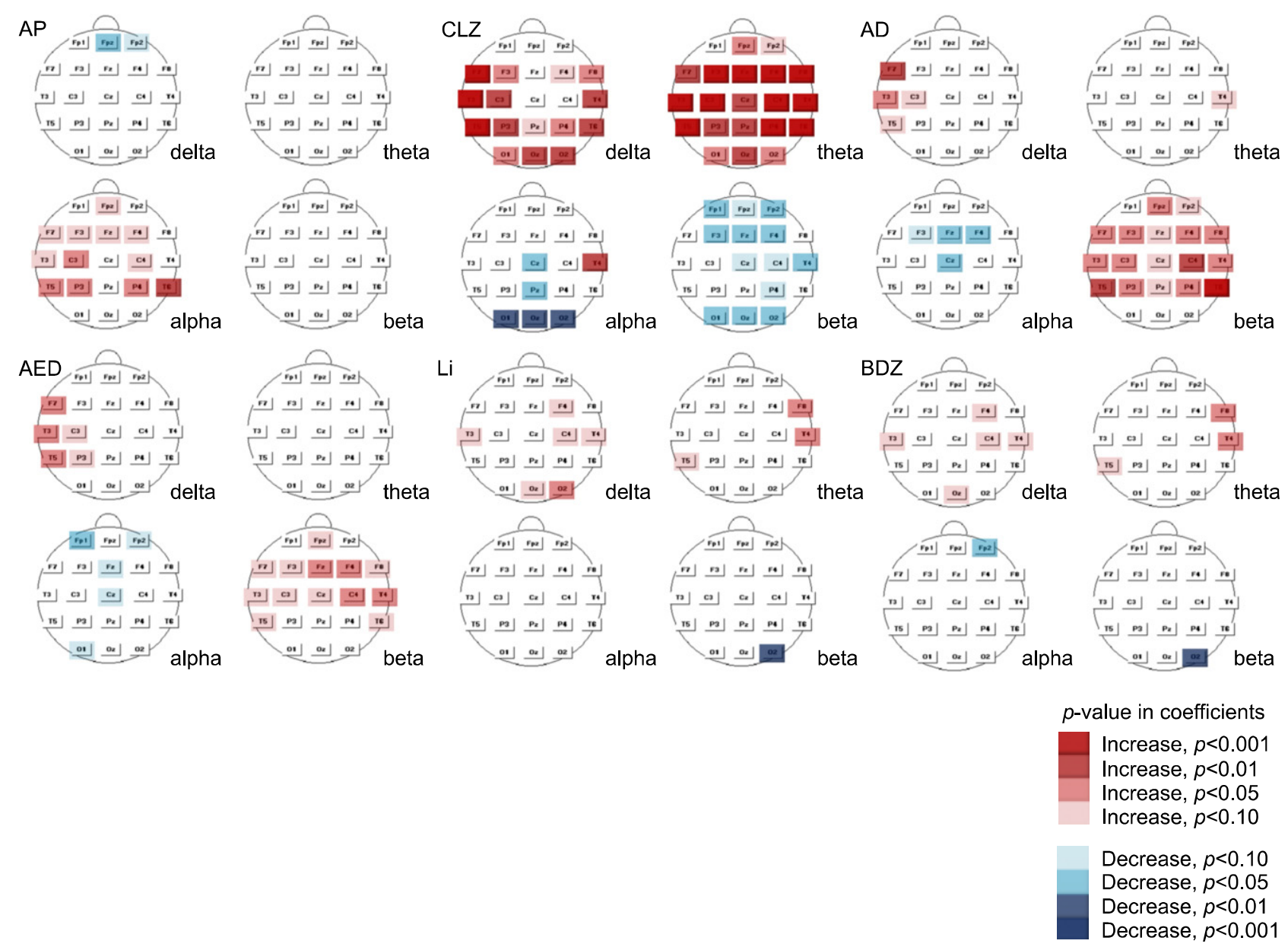

Fig. 2. Statistical probability maps according to independent samples t-tests. AP, antipsychotics other than clozapine; CLZ, clozapine; AD, antidepressants; AED, antiepileptic drugs; Li, Lithium; BDZ, benzodiazepines.

were observed among the other groups (Table 3).

\section{Statistical Probability Maps}

Fig. 1 presents statistical probability maps based on multiple regression analysis. Increased alpha relative power was observed for group AP but it was not clear in some leads (T5, P3, P4). Clozapine markedly increased slow waves, especially theta, and decreased beta in several leads. A topographical difference in alpha relative power appeared between temporal and occipital areas. Increased beta activity was the main finding for group $\mathrm{AD}$, and increased delta activity was detected in a few leads (F7, T3). An increase in beta power was also observed in group AED; several EEG leads exhibited increased delta activity, and one lead $(\mathrm{Cz})$ exhibited a decrease in alpha activity. The lithium group exhibited a clear increase in the relative power of delta and theta activity, but we did not detect any evident changes in group BDZ except for a solitary increase in alpha activity in T3.
Fig. 2 presents the results of independent sample $t$-tests. Similar to the results of the linear regression, we observed an increase in alpha relative power in group AP, but this increase was more evident than in the linear regression results. Increased delta and theta activity were still pronounced in group CLZ, with similar findings of decreased alpha activity (relatively limited to the occipital area) and decreased beta activity. We also found increased beta relative power in groups AD and AED. This analysis also revealed an increase in slow-wave activity in the lithium group, but the increase was less significant than in the linear regression results. In summary, the results of complementary $t$-tests were very consistent with linear regression analysis results, despite a few discrepancies such as the increased slow wave activity in some leads in group BDZ.

We conducted an additional analysis for clozapine. Because the correlation analysis revealed a significant correlation between groups CLZ and AP (Table 3), we needed to perform an analysis after controlling for this 

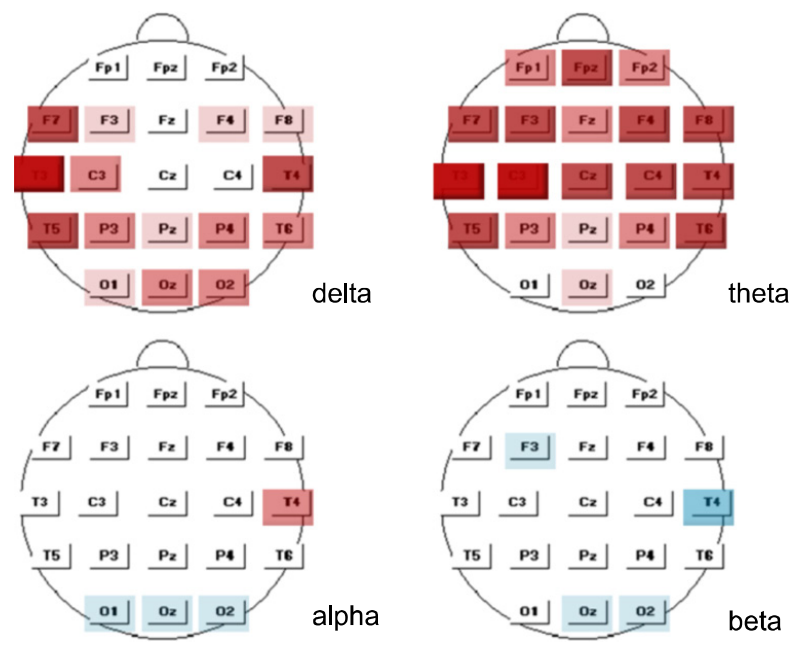

$p$-value in coefficients

Increase, $p<0.001$

Increase, $p<0.01$

Increase, $p<0.05$

Increase, $p<0.10$

Decrease, $p<0.10$

Decrease, $p<0.05$

Decrease, $p<0.01$

Decrease, $p<0.001$

Fig. 3. Relative power changes induced by clozapine in group AP AP, antipsychotics other than clozapine.

confounding factor. Therefore, we investigated mean differences in relative power between the clozapine use $(N=32)$ and non-use groups $(N=106)$ within group AP $(N=138)$ using a $t$-test. The significant increase in delta and theta relative power associated with administration of clozapine persisted after the use of other antipsychotics was controlled, as did the decreased alpha activity in the occipital area (Fig. 3). However, the decreased beta activity was less apparent than in the results of the preceding analysis, suggesting that the decrease was probably induced by the confounders.

\section{DISCUSSION}

In this retrospective, cross-sectional study, we investigated how various drugs used to treat patients with schizophrenia-spectrum disorders affected qEEG results.

Our analyses show that clozapine induced marked increases in slow-wave activity and decreased alpha activity (limited to the occipital area), which supports the findings of previous studies. ${ }^{9-12,22)}$ This result indicates that drugs concomitantly used with clozapine do not alter the effect of clozapine on EEG changes.

Our finding that antipsychotics other than clozapine tend to increase alpha activity has been reported in other studies focusing on risperidone. ${ }^{4,5)}$ However, we did not observe increases in delta and theta activity, which have been reported by studies focusing on low-potency sedating drugs. ${ }^{4,6)}$ Studies have reported little or no change in slow-wave activity among patients treated with aripiprazole, ${ }^{8)}$ amisulpride, ${ }^{7)}$ and haloperidol. ${ }^{22)}$ Thus, the effects of specific antipsychotics appear to differ. Our AP group used various antipsychotics; most of these were high-potency medications, which may have resulted in the failure to detect increases in slow wave activity.

The antidepressant group exhibited increased beta relative power, a result consistent with those of studies focusing on sertraline, ${ }^{14,23)}$ paroxetine, and fluvoxamine, ${ }^{24)}$ although there were some discrepancies in that the changes in slow-wave activity were not pronounced in our study. Our AD group was composed of various antidepressants with differing mechanisms, but because the most frequently used antidepressants were SSRIs including sertraline, our results may be grossly similar to those from SSRIs. Therefore, the results for the AD group also seemed to be grossly independent of various concomitant medications.

A few studies have investigated changes in qEEG results induced by the antiepileptic drug valproate, which is mainly prescribed for epileptic patients. ${ }^{16,25)}$ These studies have reported increases in beta activity, especially when the drug was used over a long period, which supports our findings because valproate was the antiepileptic drug most frequently used by our subjects. Several investigators have reported a consistent increase in slow-wave activity induced by other antiepileptic drugs, ${ }^{17,18)}$ which might support our finding of increased delta activity in some leads.

Our analyses revealed that lithium caused a significant increase in slow-wave activity, which has been consistently reported in previous studies. ${ }^{19,20,26)}$ This finding also suggests that concomitant use of other classes of psychotropics has little effect on changes in EEG results.

However, our results differed from those of studies reporting decreased alpha activity and increased beta activity after treatment with benzodiazepine. One possible explanation for this discrepancy is that the other medications might have acted as confounders. In fact, the correlation analysis revealed that benzodiazepine use was significantly correlated with use of other drugs such as antipsychotics. A second explanation is that an interaction with other drugs prevented us from finding a change that would have been induced if benzodiazepine were used 
alone. Most previous studies have involved the administration of a single dose of benzodiazepine to healthy subjects without any concomitant drugs, so it is possible that benzodiazepine produces different results when it is chronically used with other medication to relieve adverse events such as akathisia.

In summary, our results are generally consistent with those of previous pharmaco-EEG studies, despite some differences. The implication of these results is that human EEG results are obviously affected by psychotropic drugs and it is possible to find these changes even when they are used with other medications. Because the quantitative pharmaco-EEG can be applied to predict a patient's clinical response to psychotropic drugs, this finding is important because polypharmacy is now routinely used in clinical practice. This is the first provisional study to use statistical analysis to evaluate the effects of psychotropic drugs on quantitative EEG results.

This study had important limitations, mostly stemming from its retrospective design. We could not control for several confounding factors such as medication that the subjects had taken immediately prior to obtaining qEEG data, nor could we control for smoking and caffeine consumption, which could also influence EEG results. ${ }^{27)} \mathrm{We}$ had no information about patients' baseline EEG results or clinical conditions at the time of EEG acquisition. Pharmaco-EEG profiles are influenced by both short-term pharmacological effects of drugs and patients' long-term clinical responses to medication, ${ }^{15)}$ but we could not discriminate between them due to the lack of clinical information. However, most of our subjects were on the stable maintenance medications. Similarly the clinical information such as symptom scores, diagnostic subtypes, age of onset or total duration of illness were also lacking. Some known information such as the subjects' sex, age and the handedness were not considered for the analyses.

Despite its preliminary character, this research supports a new methodological approach to identify the quantitative EEG effects of various psychotropic drugs in clinical settings. Further prospective studies will be required to confirm our findings, and it will be necessary to correlate changes in qEEG results with each patient's clinical symptoms to evaluate their clinical significance.

\section{REFERENCES}

1. Galderisi S, Sannita WG. Pharmaco-EEG: a history of progress and a missed opportunity. Clin EEG Neurosci 2006;37:61-65.

2. Nuwer M. Assessment of digital EEG, quantitative EEG, and EEG brain mapping: report of the American Academy of Neurology and the American Clinical Neurophysiology
Society. Neurology 1997;49:277-292.

3. Itil TM, Itil KZ. Quantitative EEG brain mapping in psychotropic drug development, drug treatment selection, and monitoring. Am J Ther 1995;2:359-367.

4. Mucci A, Volpe U, Merlotti E, Bucci P, Galderisi S. Pharmaco-EEG in psychiatry. Clin EEG Neurosci 2006;37: 81-98.

5. Hughes AM, Lynch P, Rhodes J, Ervine CM, Yates RA. Electroencephalographic and psychomotor effects of chlorpromazine and risperidone relative to placebo in normal healthy volunteers. Br J Clin Pharmacol 1999;48:323-330.

6. Hubl D, Kleinlogel H, Frölich L, Weinandi T, Maurer K, Holstein W, et al. Multilead quantitative electroencephalogram profile and cognitive evoked potentials (P300) in healthy subjects after a single dose of olanzapine. Psychopharmacology (Berl) 2001;158:281-288.

7. Saletu B, Küfferle B, Grünberger J, Földes P, Topitz A, Anderer P. Clinical, EEG mapping and psychometric studies in negative schizophrenia: comparative trials with amisulpride and fluphenazine. Neuropsychobiology 1994;29: 125-135.

8. Cañive JM, Lewine JD, Edgar JC, Davis JT, Miller GA, Torres F, et al. Spontaneous brain magnetic activity in schizophrenia patients treated with aripiprazole. Psychopharmacol Bull 1998;34:101-105.

9. Galderisi S, Mucci A, Volpe U, Boutros N. Evidence-based medicine and electrophysiology in schizophrenia. Clin EEG Neurosci 2009;40:62-77.

10. Knott V, Labelle A, Jones B, Mahoney C. Quantitative EEG in schizophrenia and in response to acute and chronic clozapine treatment. Schizophr Res 2001;50:41-53.

11. Gross A, Joutsiniemi SL, Rimon R, Appelberg B. Clozapineinduced QEEG changes correlate with clinical response in schizophrenic patients: a prospective, longitudinal study. Pharmacopsychiatry 2004;37:119-122.

12. Galderisi S, Mucci A, Bucci P, Mignone ML, Maj M. Multilead quantitative EEG profile of clozapine in resting and vigilance-controlled conditions. Psychiatry Res 1996;67: 113-122.

13. Knott V, Mahoney C, Kennedy S, Evans K. Pre-treatment EEG and it's relationship to depression severity and paroxetine treatment outcome. Pharmacopsychiatry 2000;33: 201-205.

14. Siepmann M, Grossmann J, Mück-Weymann M, Kirch W. Effects of sertraline on autonomic and cognitive functions in healthy volunteers. Psychopharmacology (Berl) 2003;168: 293-298.

15. Saletu B, Anderer P, Saletu-Zyhlarz GM. EEG topography and tomography (LORETA) in the classification and evaluation of the pharmacodynamics of psychotropic drugs. Clin EEG Neurosci 2006;37:66-80.

16. Sannita WG, Gervasio L, Zagnoni P. Quantitative EEG effects and plasma concentration of sodium valproate: acute and long-term administration to epileptic patients. Neuropsychobiology 1989;22:231-235.

17. Clemens B, Ménes A, Piros P, Bessenyei M, Altmann A, Jerney J, et al. Quantitative EEG effects of carbamazepine, oxcarbazepine, valproate, lamotrigine, and possible clinical relevance of the findings. Epilepsy Res 2006;70:190-199.

18. Salinsky MC, Oken BS, Storzbach D, Dodrill CB. Assessment of CNS effects of antiepileptic drugs by using quantitative EEG measures. Epilepsia 2003;44:1042-1050.

19. Herrmann WM, Kropf D, Fichte K, Müller-Oerlinghausen B. Electroencephalographic and psychoexperimental studies in healthy subjects on lithium medication (author's transl). 
Pharmakopsychiatr Neuropsychopharmakol 1980;13:200-212.

20. Thau K, Rappelsberger P, Lovrek A, Petsche H, Simhandl C, Topitz A. Effect of lithium on the EEG of healthy males and females. A probability mapping study. Neuropsychobiology 1989;20:158-163.

21. Fitton A, Heel RC. Clozapine. A review of its pharmacological properties, and therapeutic use in schizophrenia. Drugs 1990;40:722-747.

22. Small JG, Milstein V, Small IF, Miller MJ, Kellams JJ, Corsaro CJ. Computerized EEG profiles of haloperidol, chlorpromazine, clozapine and placebo in treatment resistant schizophrenia. Clin Electroencephalogr 1987;18:124-135.

23. Saletu B, Grünberger J, Linzmayer L. On central effects of serotonin re-uptake inhibitors: quantitative EEG and psychometric studies with sertraline and zimelidine. J Neural
Transm 1986;67:241-266.

24. Saletu B, Anderer P, Saletu-Zyhlarz GM, Arnold O, Pascual-Marqui RD. Classification and evaluation of the pharmacodynamics of psychotropic drugs by single-lead pharmaco-EEG, EEG mapping and tomography (LORETA). Methods Find Exp Clin Pharmacol 2002;24 Suppl C:97-120.

25. Clemens B, Szigeti G, Barta Z. EEG frequency profiles of idiopathic generalised epilepsy syndromes. Epilepsy Res 2000;42:105-115.

26. Schulz C, Mavrogiorgou P, Schröter A, Hegerl U, Juckel G. Lithium-induced EEG changes in patients with affective disorders. Neuropsychobiology 2000;42 Suppl 1:33-37.

27. Siepmann M, Kirch W. Effects of caffeine on topographic quantitative EEG. Neuropsychobiology 2002;45:161-166. 